\title{
Optimization of Electrode Configuration in Surface-Parallel Actuated Deformable Mirrors
}

\author{
Marie Laslandes $^{a}$, Sergio Pellegrino ${ }^{a}$, John $_{\text {Steeves }}{ }^{a}$, Keith Patterson $^{b}$ \\ ${ }^{a}$ California Institute of Technology, 1200 E. California Blvd, Pasadena, CA 91125

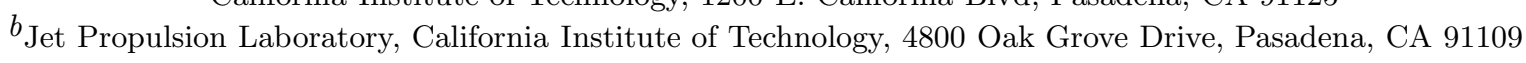

\begin{abstract}
Thin, lightweight and low-cost deformable mirrors have been recently proposed, providing a pertinent device for wavefront error correction. We present different approaches to optimize actuator arrangement. The design is optimized according to a given correction requirement, through the number of electrodes, their shape and location. A first method focuses on the compensation of a given optical aberration (astigmatism). A second method directly optimizes the correction of a set of optical modes, taking into account the voltage limitation. We will describe the optimization techniques and give some examples of applications and design performance.
\end{abstract}

Keywords: Deformable Mirror - Active Optics - Telescope - Optimization - Bimorph Mirror

\section{INTRODUCTION}

Deformable mirrors with sensing and control systems are able to correct the wavefront error in optical instruments for a variety of applications, including astronomy, ${ }^{1,2}$ high-energy laser, ${ }^{3}$ microscopy ${ }^{4}$ and ophthalmology. ${ }^{5}$ These different applications have different correction requirements: amplitude, spatial and temporal frequency of the wavefront to be corrected and precision of correction. Thus, numerous types of deformable mirror have been developed, ${ }^{6}$ based on three different actuation concepts: surface-normal actuation, surface-parallel actuation and boundary actuation. This papers focuses on surface-parallel actuated mirror, where the optical surface is bent by the in-plane stretching or contraction of an active layer laminated to the mirror face-sheet. As there is no backing structure, this solution leads to light systems, able to compensate for relatively high amplitude errors.

Active primary mirrors in earth-based telescopes have already enabled the emergence of very large apertures and, similarly, the development of larger space-based observatories will require novel active mirror technologies. ${ }^{7}$ The present paper further develops a recently proposed concept for thin deformable mirrors that promises to drastically reduce the mass, density and cost of future telescopes. ${ }^{8}$ Mirrors based on this approach are lightweight, relatively inexpensive and provide a sufficiently large shape correction capability to allow the use of nominally identical mirror segments in large segmented apertures. Accurate shape control will also allow active compensation for thermal effects and long-term material effects such as creep and aging.

The basic concept, presented in Fig. 1, consists of a laminated shell mirror that uses surface-parallel actuation. The mirror assembly is composed of a thin and stiff optical quality substrate with a layer of piezoelectric material bonded on its back-face. An electrode pattern is coated on one face of the piezoelectric layer, and a continuous ground layer is deposited on the other face. The application of an electric field to an electrode is going to retract or dilate it, causing a bending of the mirror.

Together with the mirror geometry and materials, the actuator configuration defines the system correction capabilities. The shape, position and number of actuators must be chosen according to the correction requirements. Three main types of electrode pattern are generally used (see Fig. 1). Bimorph mirrors are classically designed with a keystone layout: the actuators are arranged in rings and divided into angular domains. ${ }^{9}$ A honeycomb layout has also been used, in this case, the actuators are all identical and arranged in a hexagonal tessellation, providing a homogeneous pattern. Finally, rib-stiffened deformable mirrors and ultrathin membrane mirrors have adopted a lattice of unidirectional actuators. ${ }^{10}$

Many applications of deformable mirrors do not require the edges of the mirrors to be considered: the optical pupil is smaller than the mirror itself, making it easier to provide precise correction. However, segmented telescopes require high precision also along the edges of the mirror and this important, additional requirement is

CCC code: $0277-786 X / 14 / \$ 18 \cdot$ doi: $10.1117 / 12.2056495$ 
not adequately addressed by any of the existing actuators designs. The need for better correction along the edge of the mirror and, more generally, for maximizing the range of aberration modes that can be corrected with a given number of actuators are the main motivation of the study presented in this paper: we present two novel methods to efficiently design the active layer configuration in surface-parallel deformable mirrors.

The paper is arranged as follows. Section 2 presents the studied mirror technology and modeling. Section 3 describes a design method focused on astigmatism correction. Section 4 presents a more general method to design a pattern efficiently correcting for several optical modes. Section 5 concludes the paper.

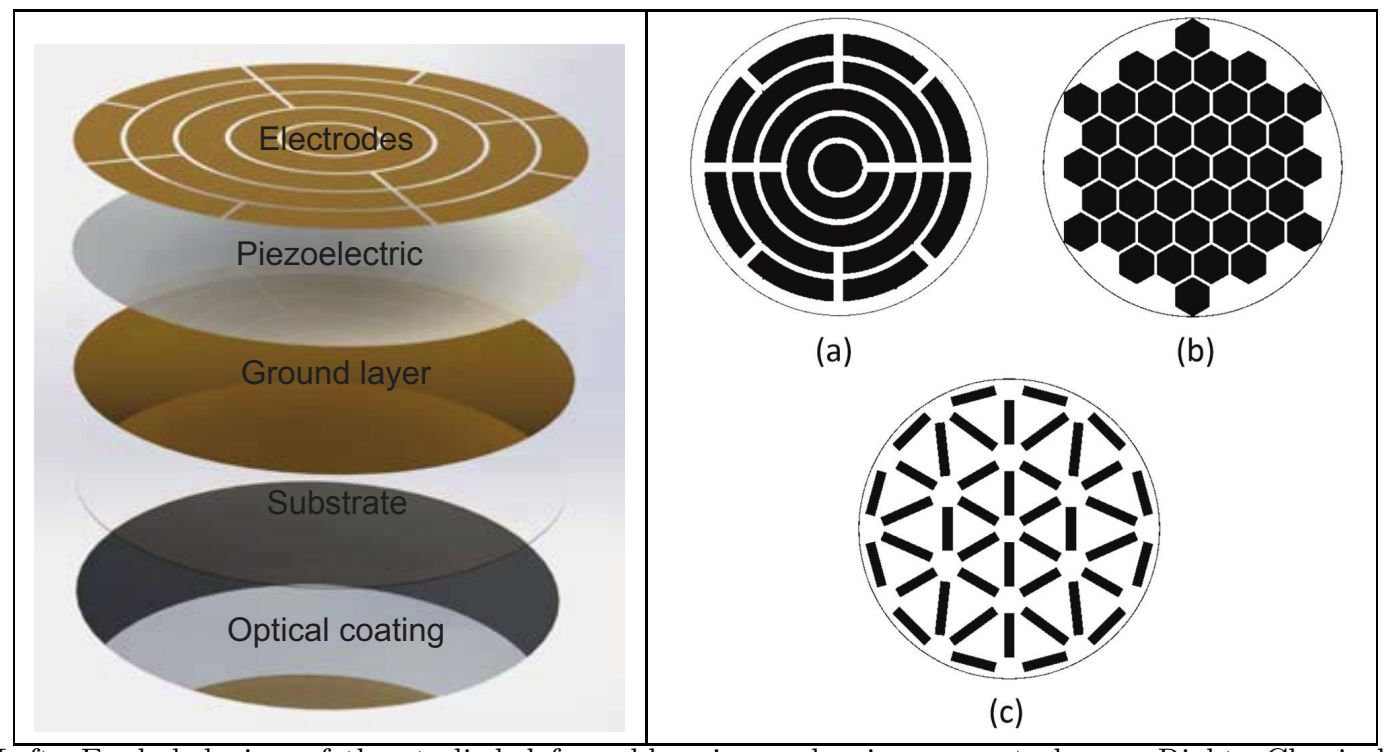

Figure 1. Left: Exploded view of the studied deformable mirror, showing separate layers; Right: Classical layouts of electrodes in surface-parallel actuated mirrors: (a) keystone; (b) honeycomb; (c) lattice.

\section{ULTRA-THIN SURFACE-PARALLEL DEFORMABLE MIRROR: CONCEPT AND MODELING}

\subsection{Technology}

As presented in Fig. 1, the mirror is composed of two main layers: a substrate and an active piezoelectric material. The piezoelectric layer covers the whole substrate, but is conceptually divided into two parts, an active part covered by the electrode and a passive part. Applying an electric field to the active part induces a mismatch strain between the substrate and the piezoelectric layer, causing the mirror to bend. ${ }^{11}$ The material and thickness of both layers are picked regarding the needed amplitude of deformation. The curvature change $k$ can be roughly estimated considering a circular system with a piezolectric layer covering the entire surface of the substrate. The deformation due to the stress mismatch is estimated through the following equation:

$$
k=6 \frac{d_{31}}{t_{p}} \Delta V \frac{\frac{t_{p}}{t_{s}^{2}} \frac{M_{p}}{M_{s}}\left(1+\frac{t_{p}}{t_{s}}\right)}{1+\left(4 \frac{t_{p}}{t_{s}}+6 \frac{t_{p}^{2}}{t_{s}^{2}}+4 \frac{t_{p}^{3}}{t_{s}^{3}}\right) \frac{M_{p}}{M_{s}}+\frac{t_{p}^{4}}{t_{s}^{4}} \frac{M_{p}^{2}}{M_{s}^{2}}},
$$

with the subscript $s$ standing for the substrate and $p$ for the piezoelectric layer. $t$ is the thickness, $\Delta V$ the applied voltage, $d_{31}$ the piezoelectric coefficient and $M=\frac{E}{1-\nu}$ the biaxial moduli ( $E$ being the Young modulus and $\nu$ the Poisson's ratio).

A rough dimensioning of the system can be done using this equation: knowing the substrate and piezoelectric materials, the thickness ratio between the two layers can be chosen with this formula. We can also note here that the thickness of the piezoelectric layer defines a limit voltage that can be applied to the system in order to 
avoid depolarization of the piezoelectric material and electrical breakdown. ${ }^{12}$

Recent studies have addressed the manufacturing process and have explored different material choices, leading to two types of mirrors. The first solution uses micro-fabrication techniques: the substrate is a wafer of singlecrystal silicon or glass and the active layer is formed from a piezo-polymer, $\mathrm{P}(\mathrm{VDF}-\mathrm{TrFE}){ }^{8}$ This approach has been demonstrated to provide high optical quality mirrors with a dynamic range of tens of micrometers. The optical diameter accessible with such a technology is limited by the manufacturing capabilities of microfabrication, typically 100-200 $\mathrm{mm}$. The second solution solves this size limit by using a simpler manufacturing process based on surface replication. The substrate is an ultra-thin carbon fiber composite shell and the active layer is made of piezo-ceramic (PZT) ${ }^{13}$ The number and thickness of single sheet carbon fiber lamina define the mechanical properties of the mirror substrate. This solution is suitable to correct wavefront errors of the order of tens of millimeters. Nevertheless, producing carbon-fiber shells of optical quality is challenging due to fiber print-through and residual stresses resulting from the curing process.

\subsection{Finite Element Model}

The mirror assembly is modeled with the finite element package Abaqus standard. ${ }^{14}$ The mirror model is constructed using a composite thin shell structure and meshed with thermoelastic thin shell elements S4T. Several layers are defined within the shell thickness, representing the substrate and the active material. Each layer is characterized by its thickness and mechanical properties. Thermally induced strains are used to simulate the piezoelectric strains, hence a temperature field is applied as a substitute for the electric field and the thermal expansion coefficient for the $d_{31}$ coefficient of the piezoelectric material. The thermal expansion coefficient is scaled so that a temperature variation of $1 \mathrm{~K}$ is equivalent to the application of $1 \mathrm{~V}$ across the faces of the piezoelectric layer.

The actuators are defined by dividing the shell into active and passive sections. Passive sections correspond to part of the mirror that are not actuated, their thermal expansion coefficient is set to 0. Each active section corresponds to one actuator, it can be controlled independently from the other, with a given temperature variation. Once the different sections are defined, a temperature field is applied to the model and the finite element analysis can be run. The displacements of each node of the model are recovered as an output. Detailed estimates can then be obtained, for any chosen electrode patterns.

\subsection{Performance Prediction}

The system behavior is characterized through its influence functions. The influence function $I F_{i}$ is defined as the wavefront error resulting from a reflection on the mirror when a unit command is send to the actuator $i$, the other actuators being at rest. A displacement vector is recovered from finite element analysis and the induced wavefront error is twice this mirror deformation. All the influence functions of a mirror are regrouped in an influence matrix $M$. To correct a wavefront error $P$, an adequate vector voltage $V$ must be send to the mirror. The residual wavefront error, obtained after reflection on the deformed mirror is given by:

$$
P_{\text {res }}=P-M V \text {. }
$$

Thus, the optimal set of voltages, minimizing the residual error, is determined by projecting the wavefront error to be corrected onto the influence matrix $M$. As explain in Sec. 2.1, the piezoelectric material properties limit the voltages to a value $V_{l}$. To take this physical limit into account, the voltages are computed using a constrained least square algorithm: ${ }^{15}$

$$
V=\min _{X}\left(\frac{1}{2}\|M X-P\|_{2}^{2}\right), \text { with }-V_{l}<V<+V_{l},
$$

The performance of a given system for the correction of a wavefront error $P$ is generally given by the root mean square (RMS) amplitude of the residual wavefront map.

A wavefront error $P$ can be decomposed onto a Zernike polynomials basis $Z: P=\sum_{i} a_{i} Z_{i}$, where $Z_{i}$ is the $i^{\text {th }}$ mode of the base (as described in Noll ${ }^{16}$ ) and $a_{i}$ its RMS amplitude. One way to characterize a deformable 
mirror performance is then to study the correction of specified Zernike modes. The correction performance of one mode $P_{i}=a_{i} Z_{i}$ can be given by both its correctability and stroke. Its correctability, $c_{i}$, is the ratio between the RMS amplitude of the wavefront error before and after correction, obtained without voltage limit:

$$
c_{i}=\frac{\left\|P_{i}\right\|_{2}}{\left\|P_{i}-M V_{t h}\right\|_{2}}
$$

with $V_{t h}$ given by Eq. 3 with no bounds on $V$.

And its stroke, $s_{i}$, is the maximum amplitude of the mode that can be corrected without saturating any actuators:

$$
s_{i}=\frac{V_{l}}{\max \left(V_{t h}\right)} a_{i} .
$$

While the correctability depends only on the geometry of the electrode pattern, the stroke depends on the mirror geometry and material.

\section{OPTIMIZATION OF ASTIGMATISM CORRECTION}

Third-order astigmatism is one of the most important aberration modes in an optical system: it is a significant component of the initial shape distortion of mirrors and one of the first off-axis aberrations induced by misalignment. ${ }^{17}$ Hence, deformable mirror designs need to be particularly efficient in the correction of this mode and, because its magnitude is often large, a significant stroke is also needed. This section presents the design of actuator patterns optimized for astigmatism3 correction.

The approach that has been chosen begins by identifying a single electrode system that is optimized to directly generate the required correction mode. Subsequently, additional electrodes are considered, both to improve the initial single mode correction performance and also to allow the correction of other aberrations.

\subsection{Single Electrode Approach}

As an initial step, consider the simplest problem of designing a single electrode system to correct for the particular astigmatism 3 shape error. The shape and position of the electrode are optimized by coupling finite element analysis with a minimizer suitable for non-convex problems. Here we use an algorithm that performs a global search of the design space: the covariance matrix adaptation evolution strategy algorithm (CMAES). ${ }^{18}$

Assuming the actuator to have a singly-connected shape, it can be defined in polar coordinates $(r, \theta)$ by a set of points equally spaced in the angular direction, see Fig. 2. The radial positions of these contour points are the optimization algorithm variables. At each trial, a finite element model of the mirror and actuator is created and the system performance is characterized by computing its correctability and stroke (Eqs. 4 and 5). In order to maximize both quantities, the following multi-objective function $f$ is defined:

$$
f=\lambda_{1} c_{a s t m}+\lambda_{2} s_{a s t m}
$$

$\lambda_{1}$ and $\lambda_{2}$ being weights allocated to the two quantities, depending on specific correction requirements.

The optimal shape of the electrode is then obtained by maximizing the value of $f$ through changes in the actuator shape.

Note that the required correction mode and the electrode shape must have the same type of symmetry. Astigmatism3 has two planes of mirror symmetry, hence only a quarter of the basic electrode shape needs to be determined and its full geometry is constructed with two mirror symmetry operations. 


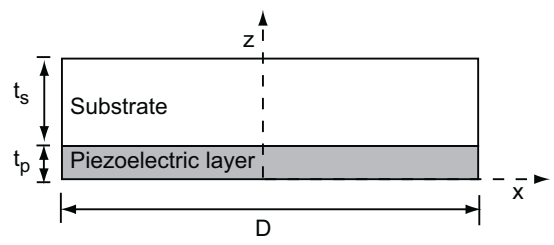

(a)

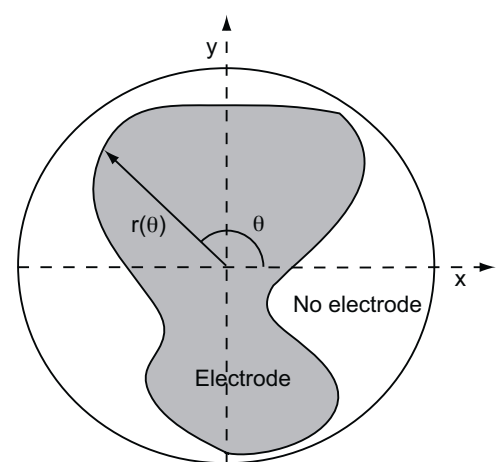

(b)

Figure 2. Piezoelectric layer concepts: definition of electrode shape for single-actuator optimization.

The optimization method is applied to a $100 \mathrm{~mm}$ diameter thin glass mirror. The mirrors are flat, composed of a $200 \mu \mathrm{m}$ layer of glass and a $20 \mu \mathrm{m}$ layer of $\mathrm{P}(\mathrm{VDF}-\mathrm{TrFE})$, and the limit voltage is $500 \mathrm{~V}$. The optimization problem was formulated as described in Eq. 6, biasing the solution towards higher correctability and lower stroke, by assigning $\lambda_{1}=10$ and $\lambda_{2}=1$.

The results of the shape optimization for a single electrode are presented in Fig. 3. The shape of the electrode was calculated by computing the profile $r(\theta)$ at 6 points located in one quadrant. The optimal shape is a bow-tie shape and the obtained correction is close to a cylindrical deformation. For an initial astigmatism3 error of $1 \mu \mathrm{m}$ RMS, the residual total wavefront error is $1.587 \mu \mathrm{m}$ RMS, reduced to $0.378 \mu \mathrm{m}$ RMS after removal of the focus aberration.

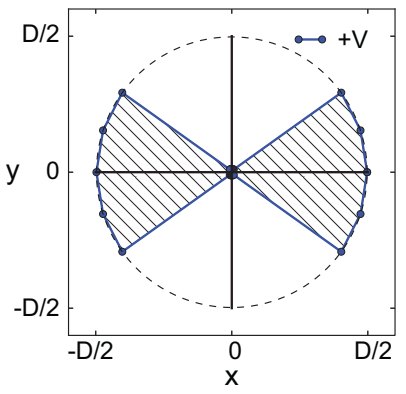

(a)

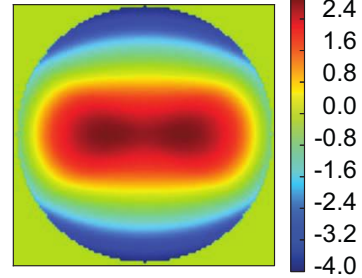

(b)

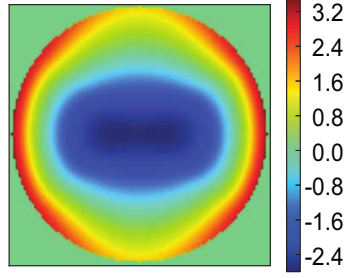

(c)

Figure 3. Optimized electrode shapes and system performance for the correction of $1 \mu \mathrm{m}$ of astigmatism3 (without limit voltage): (a) single-actuator configuration; (b) correction, including focus aberration (1.875 $\mu \mathrm{m}$ RMS); (c) residual wavefront error (1.587 $\mu \mathrm{m}$ RMS). Units: $[\mu \mathrm{m}]$.

\subsection{Twin Actuators}

As we have just seen, a single electrode system tends to induce a curvature mode, hence generating a large amount of focus aberration. ${ }^{19}$ A second actuator can be introduced to reduce the focus aberration and thus improve the overall correction capability. One solution consists in using two electrodes with identical shape, but rotated of $\pi / 2$ and actuated with equal and opposite voltages. The focus change induced by the first electrode is then directly suppressed by the focus change induced by the second electrode. The specific rotation angle of $\pi / 2$ ensures that the correction mode generated by the second actuator has the same orientation as that generated by the first actuator, it provides double the correction amplitude of each single actuator.

The electrode pattern is based on the contour shape $r(\theta)$, which is optimized for optimal performance of the twin-actuator system. The correction provided by the first-actuator is predicted by finite element analysis and the effect of the second actuator is simply obtained by superposing a rotated deformation map to the original one. The combined deformation is used in the optimization process. 
A twin-actuator system was designed, considering the same system and objective function than for the single electrode. The basic electrode shape is presented in Fig. 4, it resembles an ellipse. Overall, the twin-electrode pattern consists of a large central part defined by the intersection between the two ellipses, and subjected to a voltage of zero, surrounded by four crescent-shaped regions alternately subject to positive and negative voltages of equal magnitude. This system is able to correct astigmatism3 with a correctability of 10 and a stroke of $3 \mu \mathrm{m}$ RMS.

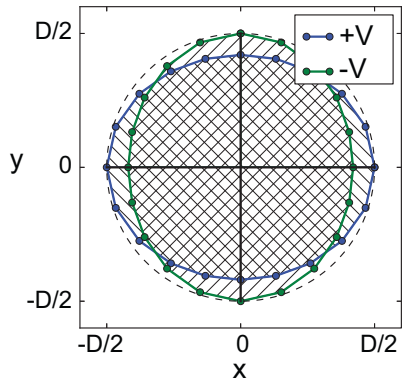

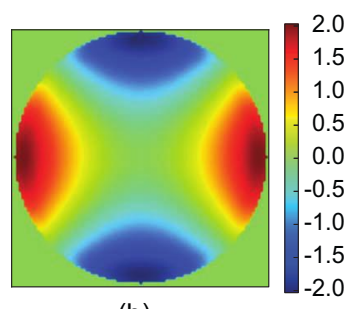

(b)

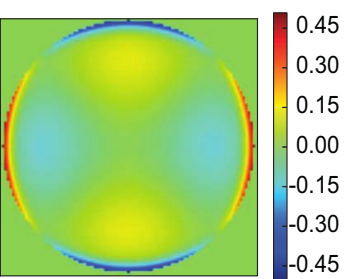

(c)

(a)

Figure 4. Optimized electrode shapes and system performance for the correction of $1 \mu \mathrm{m}$ of astigmatism3 (without limit voltage): (a) twin-actuator configuration; (b) correction (1.002 $\mu \mathrm{m}$ RMS); (c) residual wavefront error (0.104 $\mu \mathrm{m}$ RMS). Units: $[\mu \mathrm{m}]$.

The size of the four edge actuation zones depends on the weights given to stroke and correctability, when defining the optimization function, but the overall pattern is general. For simplicity the basic electrode shape is approximated by an ellipse and described by the lengths of the horizontal and vertical semi-axes. The ratio between the major axis (which is always equal to the mirror diameter) and the minor axis of the ellipses drives the system performance. The performance trends have been analyzed through finite element models with different ellipse axis ratio; the results are shown in Fig. 5. A configuration with an axis ratio close to 1, which has very narrow actuation zones is the most efficient in correcting astigmatism3 but is limited in terms of stroke. On the other hand, a configuration with a smaller axis ratio leads to bigger actuators and significantly improves the stroke, at the expense of the correctability.

Figure 5 also shows that there is a significant impact in reducing the optical pupil diameter. Because the residual error is largest near the edges of the mirror, a smaller pupil leads to a better correctability.

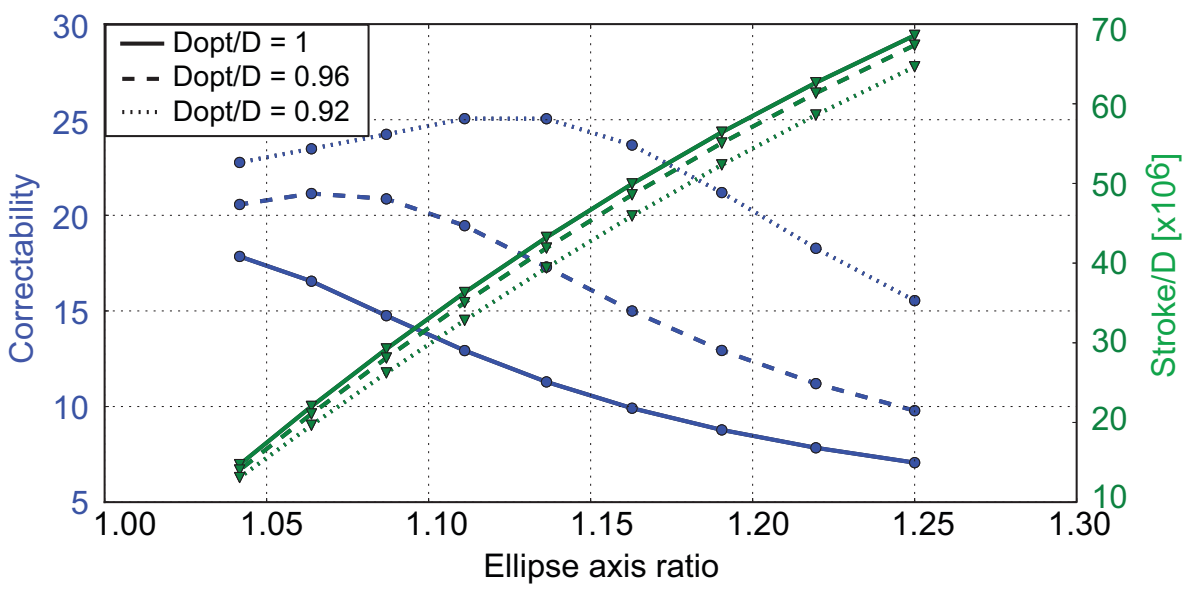

Figure 5. Evolution of correctability and stroke with ellipse and pupil dimensions, for astigmatism3 correction provided by systems with twin elliptical actuators (see Fig. 4). 


\subsection{Additional Actuators}

The twin-actuator system leaves a residual wavefront error mainly composed of higher-order astigmatism. To compensate for this residual error, an additional twin-actuator system is added, whose shape is again optimized. The optimization method remains unchanged, except for the introduction of a boundary condition on the shape of the contour of the actuator, to avoid any overlap between the electrodes. The common shape of the two additional orthogonal electrodes is determined by solving the same optimization problem than previously.

Figure 6 presents the results for a system consisting of two sets of nested twin-actuators. The shape of the inner electrodes is also closely approximated by an ellipse; the major axis of the inner ellipse matches the minor axis of the outer ellipse, leading to a maximum area of active material. By introducing the inner twin actuator, the correctability is increased by a factor of 2 .

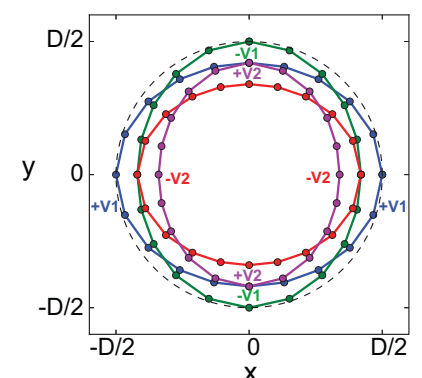

(a)

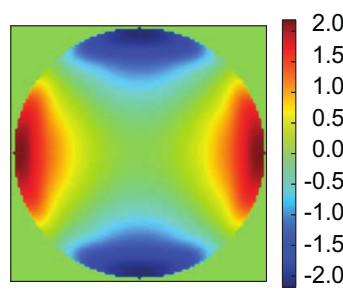

(b)

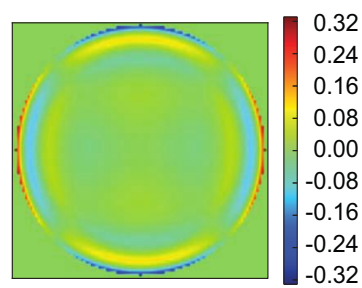

(c)

Figure 6. Optimized electrode patterns and system performance for the correction of $1 \mu$ m of astigmatism3 (without limit voltage): (a) two sets of twin actuators; (b) correction (0.993 $\mu \mathrm{m}$ RMS); (c) residual wavefront error $(0.053 \mu \mathrm{m}$ RMS).

Further improvements in the correction performance can be achieved by adding further sets of nested twinactuators, each lying inside the previous ones, whose shape is generated in the same manner. The outcome is an actuator system with several nested rings of orthogonal ellipses. Moreover, to generate astigmatism3 correction in any orientations, the electrode pattern needs to be rotated of $\pi / 4$ and added to the initial pattern. Since all these actuator systems are built on the same piezoelectric layer, each intersection between the two patterns defines a new actuation zone. For example, Fig. 7 shows an actuation system consisting of two rotated pattern of four nested rings of orthogonal ellipses. The final electrode pattern consists of 129 independent actuators, providing an highly efficient correction of astigmatism in both $x$ and $y$ directions.

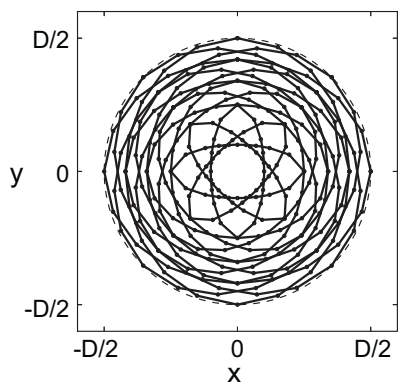

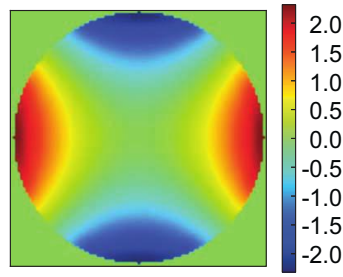

(b)

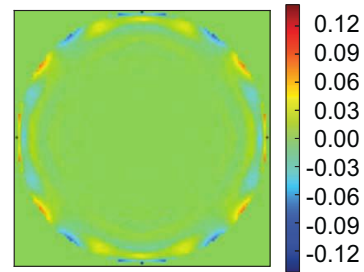

(c)

(a)

Figure 7. Optimized electrode patterns and system performance for the correction of $1 \mu$ m of astigmatism3 (without limit voltage): (a) four sets of twin actuators plus four additional sets at $45^{\circ}$ resulting in 129 actuation zones; (b) correction (0.994 $\mu \mathrm{m}$ RMS); (c) residual wavefront error (0.014 $\mu \mathrm{m}$ RMS). Units: $[\mu \mathrm{m}]$.

Compared to the configuration in Fig. 6, the correctability of the 129-actuators pattern is improved by a factor of almost 10, however the stroke is reduced by a factor of about 5 . The actuators defined by the edge of the mirror and by the outermost ellipses are tiny. The required voltage is 100 times larger in these actuators than 
for the innermost actuators in the pattern. But, even when some of the actuators are saturated, the mirror is still able to perform further correction, although the performance of the system is degraded. The evolution of the amplitude of the residual error (output) with the initial error amplitude (input) was studied and is presented in Fig. 8. For small inputs there are no saturated actuators and hence the output varies linearly with input; the slope of the output/input graph corresponds to the system correctability, which has an initial value of 71 . When some of the tiny edge actuators reach saturation, the correction performance is slightly degraded as the slope of the output/input graph is increased. However, the overall performance remains quite good, with a correctability of 60 . For input amplitudes of around $1.3 \mu \mathrm{m}$ RMS, the edge crescent actuators also begin to reach saturation and from this point on there is a significant decrease in correctability.

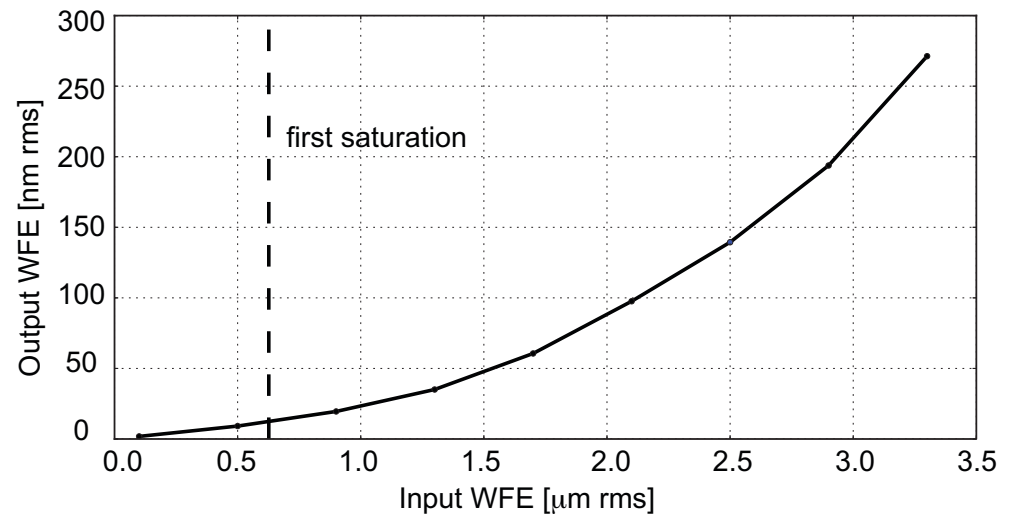

Figure 8. Performance of 129-actuator system: evolution of residual wavefront error with initial amplitude of astigmatism3 (considering a pupil of $98 \%$ of the total mirror diameter).

In addition to providing an efficient correction of astigmatism3, the 129-actuator layout can be used to correct other types of aberrations. Its performance in correcting the first 25 Zernike modes has been computed and is presented in Fig. 9. The evolution of the correctability with Zernike modes of increasing order is most satisfactory; for a pupil diameter of $98 \mathrm{~mm}$ the correctability is higher than 10 for all but two modes. For a pupil diameter of $90 \mathrm{~mm}$ the correctability is higher than 30 for the first 20 modes. The computed stroke appears to be quite low, but recall that the stroke has been defined at the point of first saturation. It has already been shown that, beyond first saturation, much larger aberration amplitudes can be corrected without significant impact on correctability.

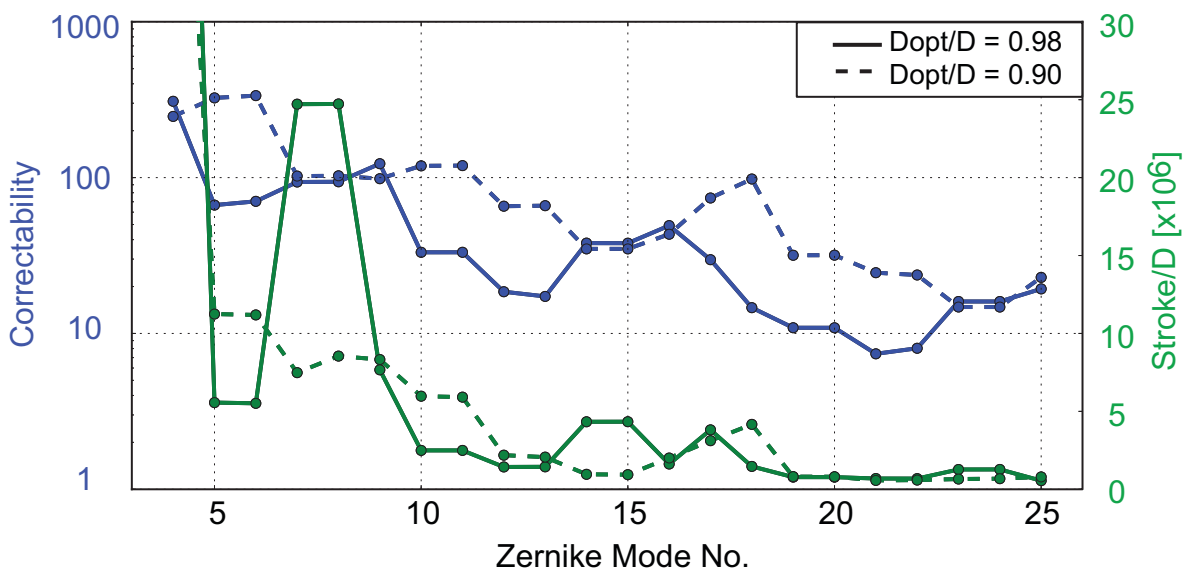

Figure 9. Performance of 129-actuator system: correctability and stroke of first 25 Zernike polynomials for two pupil sizes. 


\subsection{Manufacturing Constraints}

The 129-actuator mirror, although optimized for astigmatism3 correction, is able to efficiently correct the first 25 Zernike polynomials, making this design interesting for many applications. However, there are some practical limitations that need to be taken into account before considering its physical implementation. First, each electrode has to be large enough that a physical connection to the voltage controller can be built. It is assumed here that the electrode surface must provide a minimum contact area of $1 \times 1 \mathrm{~mm}^{2}$. Second, the electrodes must be physically separated for insulation purposes. A minimum inter-electrode distance of $0.5 \mathrm{~mm}$ is assumed here. Finally, to avoid shorting across the edges of the mirror, the electrodes cannot extend to the edge of the mirror, hence a clear annulus with a radial width of $1.5 \mathrm{~mm}$ minimum is assumed.

These constraints induce a performance reduction that can be characterized with finite element analysis. The model of the optimized mirror was hence modified to incorporate the manufacturing constraints described above. Introducing the clear edge annulus has the effect of removing the eight tiny electrodes near the edge, reducing the number of actuators to 121. This loss is mostly affecting astigmatism and trefoil correction but this effect can be mitigated by a reduction of the pupil diameter. The introduction of inter-actuator gaps affects all modes roughly in the same way. Overall, due to the manufacturing constraints, the correctability is decreased in average by $30 \%$ and the stroke by $20 \%$.

It is interesting to explore the trade-off between performance and total number of independent actuation channels. Starting from the 121-actuator pattern, the number of actuators was reduced by grouping together neighboring actuators and the performance of simplified mirror designs was computed. The four axes of symmetry of the initial pattern were maintained throughout this process and the edge actuators were not modified as they are essential for astigmatism 3 correction. The evolution of the actuator pattern from 121 to 41 actuators is presented in Fig. 10. The performance of the mirror decreases rather slowly when the 121 actuators are reduced to 57: in average, the correctability is decreased by only $20 \%$ although the number of actuators is more than halved. If the number of actuators is further reduced, the rate of decrease in performance suddenly increases; there is a further reduction of $20 \%$ when going from 57 to 41 actuators. These preliminary results can be used to guide the selection of the best design choice for a given application.

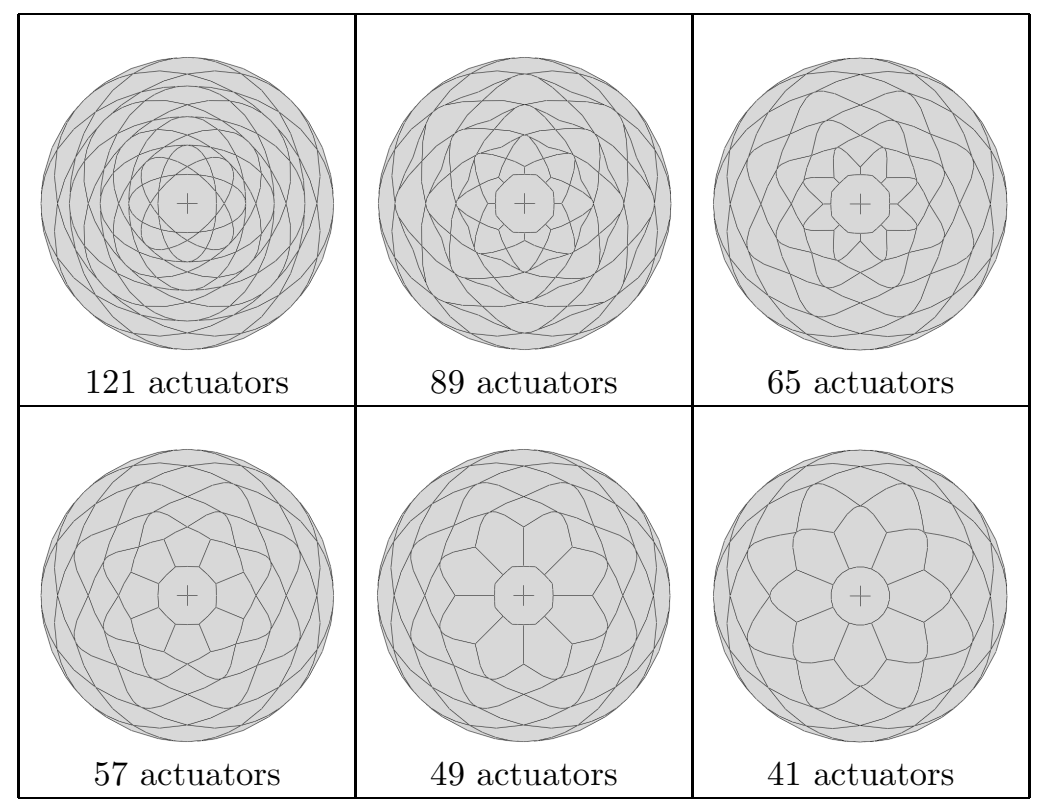

Figure 10. Designs with decreasing numbers of actuators. 
In conclusion, we have described a method allowing the design of an actuator pattern efficiently correcting for astigmatism3. As it presents numerous actuators it also provide efficient correction of other Zernike modes. The 41-actuator mirror was manufactured and tested with the techniques described in Patterson and Pellegrino ${ }^{8}$ (see Fig. 11). Its experimental characterization has validated this optimization method.

Finally, note that the method has been applied here for astigmatism3 correction on glass mirror but it can be adapted to any surface-parallel actuated mirror technology and to any figure error presenting at least two planes of mirror symmetry.

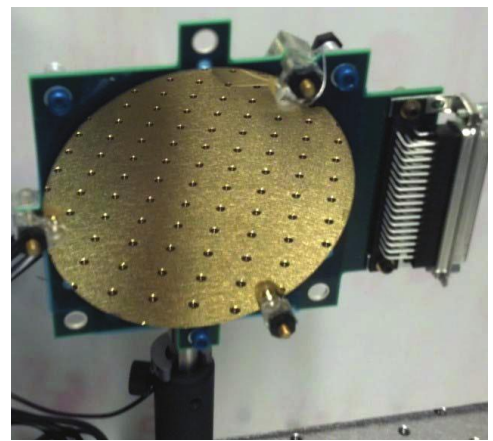

(a)

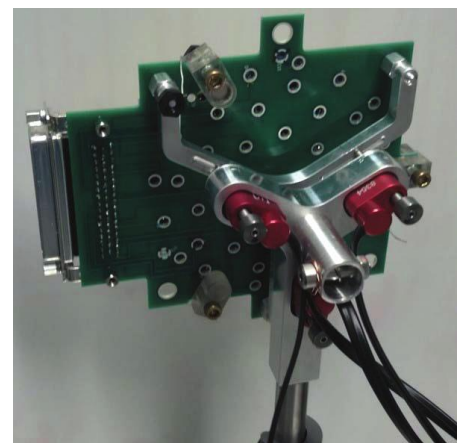

(b)

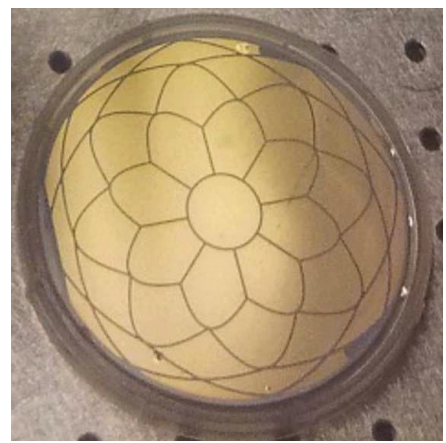

(c)

Figure 11. Prototype of a 41-actuator thin deformable glass mirror: (a) Front view of the mounted mirror showing reflective surface and three mounting points ; (b) Rear view of the mounted mirror showing electronic board and gimbal; (c) Electrode pattern.

\section{OPTIMIZATION OF THE CORRECTION OF SEVERAL MODES}

A more general method has then been developed, it optimizes the electrode pattern directly considering the correction of several error modes and it takes into account actuator saturation in the design process. The approach consists in starting with a mirror design containing numerous small actuators and grouping them to form new larger actuators. A finite element model of the initial mirror is created and an optimization algorithm is used to find an efficient grouping, regarding the set of aberrations to be corrected.

\subsection{Problem Formulation}

The goal of the optimization is to design an actuator pattern correcting for the best a set of $N_{Z}$ specified optical modes $\left\{P_{i}=a_{k} Z_{k}\right\}, i$ going from 1 to $N_{Z}, k$ being the numbers of the $N_{Z}$ considered Zernike modes and $a_{k}$ their associated RMS amplitudes.

The correction of each mode is considered independently and the objective is to minimize the quadratic sum of each mode residual amplitude. The merit function $f$ is this time:

$$
f=\sqrt{\sum_{i=1}^{N_{Z}}\left\|P_{\text {res }, i}\right\|_{2}^{2}}=\sqrt{\sum_{i=1}^{N_{Z}}\left\|P_{i}-M V_{i}\right\|_{2}^{2}} .
$$

The quantity to be optimized is then $M V_{i} . M$ is the system influence matrix. The core of the process is to find an optimal matrix, through the optimization of the actuator arrangement. $V_{i}$ is the voltage vector allowing the correction of the mode $P_{i}$ with the considered electrode pattern, computed through Eq. 3.

This approach ensures that the system performance will not be limited by actuator saturation. However, the actuator saturation is only taken into account for the correction of each individual mode and the objective function corresponds to the residual error that would be obtained if the system has to perform a correction of all the input error modes in the same time, at their maximal amplitude, provided no additional actuator saturation. This is a valid approximation because such a case is unlikely and the voltages required for the correction of one mode could balance the values required for another mode.

In this problem, the importance of one error mode compared to the others in the design process is given by the 
required amplitudes: the amplitude ratios between the different modes act as weights in the optimization.

Once the correction requirements are defined, a deformable mirror geometry can be chosen and a finite element model created. The piezoelectric layer is patterned with $N_{1}$ small square actuators, that we call pixel actuators (see Fig 12). These pixel actuators are organized in a regular grid, defined by its sampling $s$. The $N_{1}$ influence functions of the system are computed, they form the pixel influence matrix $M_{1}$, which entirely characterizes the mirror mechanical behavior.

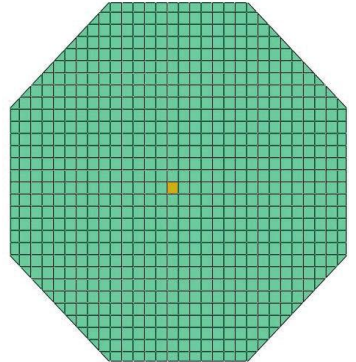

(a)

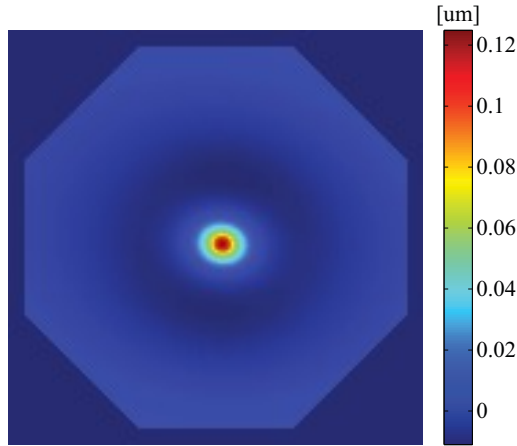

(b)

Figure 12. (a) Finite element model of a 8-ply carbon shell mirror with 756 pixel actuators, the highlighted pixel actuator is actuated in the finite element analysis. (b) Pixel influence function of the actuator highlighted in (a).

The last input is the number of independent actuators wanted on the final mirror design: $N_{2}<N_{1}$. The goal of the process is to optimally pattern the active layer with this given number of electrode: the optimization consists in grouping the initial $N_{1}$ pixel actuators into $N_{2}$ groups.

The pixel actuators are grouped through a Voronoi diagram. ${ }^{20}$ A set of $N_{2}$ points $\left(x_{v}, y_{v}\right)_{j}$ is defined and they are used to divide the mirror space into $N_{2}$ regions. The points are called sites and the regions are Voronoi cells. Each cell is associated to one site and contains all points closer to that site than to any other. A pixel actuator $i$ is defined by the coordinates of its center $\left(x_{a c t}, y_{a c t}\right)_{i}$ and will be allocated to the group defined by the Voronoi cell containing this point (Fig 13).

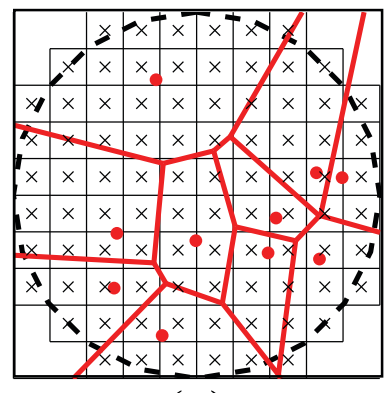

(a)

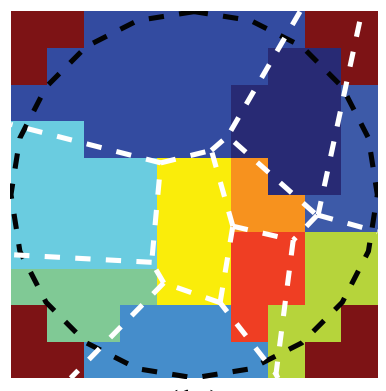

(b)

Figure 13. Grouping actuators into Voronoi cells: (a) 10 Voronoi cells (associated to 10 sites) shown on top of an initial pixel actuator grid ; (b) Corresponding actuators grouping (each color represents one electrode).

This way of grouping ensures that all pixel actuators of a same group are adjacent and that every pixel actuators belong to one group and one group only. However, the use of a Voronoi diagram constraints the shapes of the grouped electrodes to convex polygons. It is not possible to consider a complete design space with this method, but it allows to explore in a relatively quick way an extended design space. Moreover, the grouped electrodes 
have pixelized contours. This might have some effects on the system performance but it can be minimized by starting with an adequate sampling of pixel actuators.

As the system behavior is considered linear, the response of several actuator can simply be added. So, once each pixel actuator is allocated to a group, the combined influence function can be computed, by summing all the pixel influence functions of the group. The combined influence function define a new influence matrix $M_{2}$, which characterizes the behavior of the system composed of the $N_{2}$ grouped electrodes: the correction performance for this new configuration can be computed, as explained in Sec. 2.3 and the value of the objective function deduced (Eq. 7).

The optimization algorithm tries a number of pixel actuators grouping in order to converge to a configuration minimizing the objective function. At each iteration, the algorithm tries a new set of Voronoi sites $\left(x_{v}, y_{v}\right)_{j}$, there are then $2 N_{2}$ variables. In order to reduce the space of search, bounds are applied to the location of the sites: they cannot be outside the mirror surface. Moreover, as before, the number of variables can be reduced by considering symmetry of the system and of the correction modes. For instance if there are two planes of mirror symmetry, the pattern can be searched on one quarter of the mirror and defined elsewhere using two mirror symmetries. This problem is non convex and present a large number of variables. Once again, the covariance matrix adaptation evolution strategy algorithm (CMAES) is used. ${ }^{18}$

The advantage of this method is that there is no need to create a finite element model for each analyzed mirror design. Once the pixel influence functions are recovered, the optimization is performed without going back to the model, which allows a significant gain of time. However, we have seen that the resulting electrodes shapes will have pixelized contours. At the end of the process, one can envisage to modify the optimized pattern in order to smooth the actuators edge, to make it more representative of a realisable pattern. But for a first characterization of the performance, the current method is enough. Furthermore, the introduction of manufacturing constraints would automatically remove the pixelization.

\subsection{Simple Example: Astigmatism3 Correction}

Developments are currently done on carbon shell mirrors. ${ }^{21}$ The improvement of their optical quality will be achieved by working on the manufacturing process and on the electrode pattern. The developed design method is then applied to this type of mirror. The considered mirror prototype is octagonal and measures $16 \mathrm{~cm}$ corner to corner. It is nominally spherical, with a radius of curvature of $2.5 \mathrm{~m}$. It is an assembly of 8 ply of carbon fiber composite T800. The carbon shell substrate is $240 \mu \mathrm{m}$ thick. A $125 \mu \mathrm{m}$ thick PZT plate is bonded on the backface of the substrate. The maximum voltage allowable with such a thickness is $100 \mathrm{~V}$. After manufacturing, the mirror presents some deviations from its nominal spherical shape that the actuators must correct efficiently. As a first approximation, this initial error is assumed to be a pure astigmatism3 with an amplitude of $130 \mu \mathrm{m}$ RMS: that is the our optimization goal.

A finite element model of the corresponding mirror is created. As shown on Fig 12, the active layer is patterned with 756 pixel actuators, which are $5 \times 5 \mathrm{~mm}$ (except for the ones on the edges that are only a part of a square). The assembly is meshed in order to have 25 elements per actuator. Considering the mirror shape, the ply assembly symmetry and the symmetry of the astigmatism error, it is possible to run the optimization on one quarter of the mirror. Once the pixel actuators are grouped on this quarter, the electrodes on the rest of the mirror are constructed with mirror symmetries.

A 8-actuator system is designed in order to correct the initial error (130 $\mu \mathrm{m}$ RMS of astigmatism3). As, the grouping is done on one quarter of the mirror, there is only 2 electrodes shapes to be optimized, hence 4 variables. The optimized pattern is presented on Fig 14(a), together with the electrodes associated voltages. Results similar to the twin-actuator configuration of the previous method are recovered. The optimized pattern is composed of four small actuators along the mirror edges and four bigger central actuators. The position of the edge actuators corresponds to the location of lobes of the astigmatism3 figure and the voltages required on these actuators have identical values $(50 \mathrm{~V})$ and opposite signs. The voltages of the four central actuators are really small: the correction is done mainly with the four edge actuators. The obtained residual error is $7.7 \mu \mathrm{m}$ RMS: the system 
has been efficiently designed, it corrects $94 \%$ of the initial error.

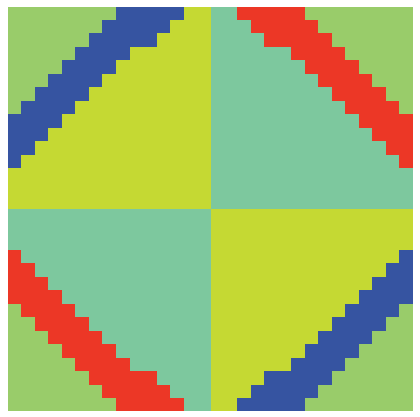

(a)

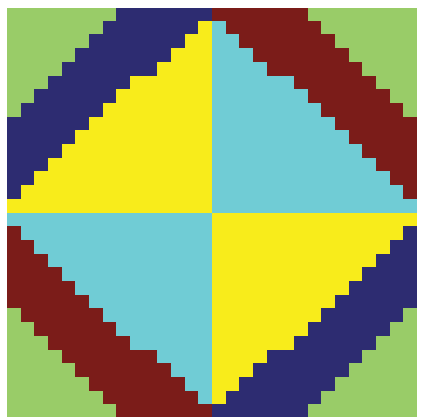

(b)

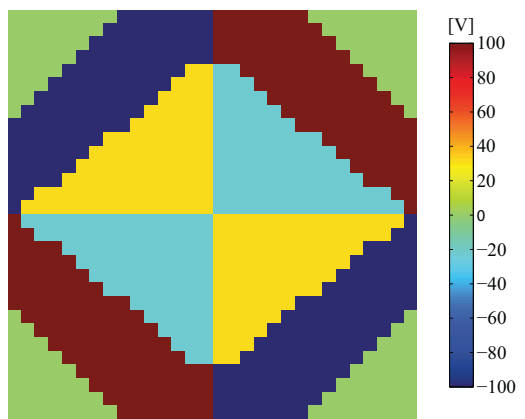

(c)

Figure 14. Patterns optimized for different input amplitude of astigmatism3 error and associated voltages: (a) Results for the optimization of $130 \mu \mathrm{m}$ RMS ; (b) Results for the optimization of $300 \mu \mathrm{m}$ RMS ; (c) Results for the optimization of $400 \mu \mathrm{m}$ RMS.

Once the initial shape error is corrected, additional astigmatism3 error might appears due to environment variations or misalignments. The evolution of the residual error, obtained with the studied pattern, depending on the amplitude of the initial astigmatism3 error is studied, it is the blue curve on Fig 15. Three different functioning domains are observed in this curve. First the residual amplitude varies linearly with the input amplitude. Then for an initial error of $200 \mu \mathrm{m}$ RMS, the voltages on the edge actuators reach the limit value, they cannot be more actuated, so the central actuators take over a part of the correction. The slope of the curve changes at this point, the correction is not as efficient as before: only $83 \%$ of the initial error is corrected. Then, for an error of $500 \mu \mathrm{m}$, the central actuators also reach saturation and no further correction can be performed.

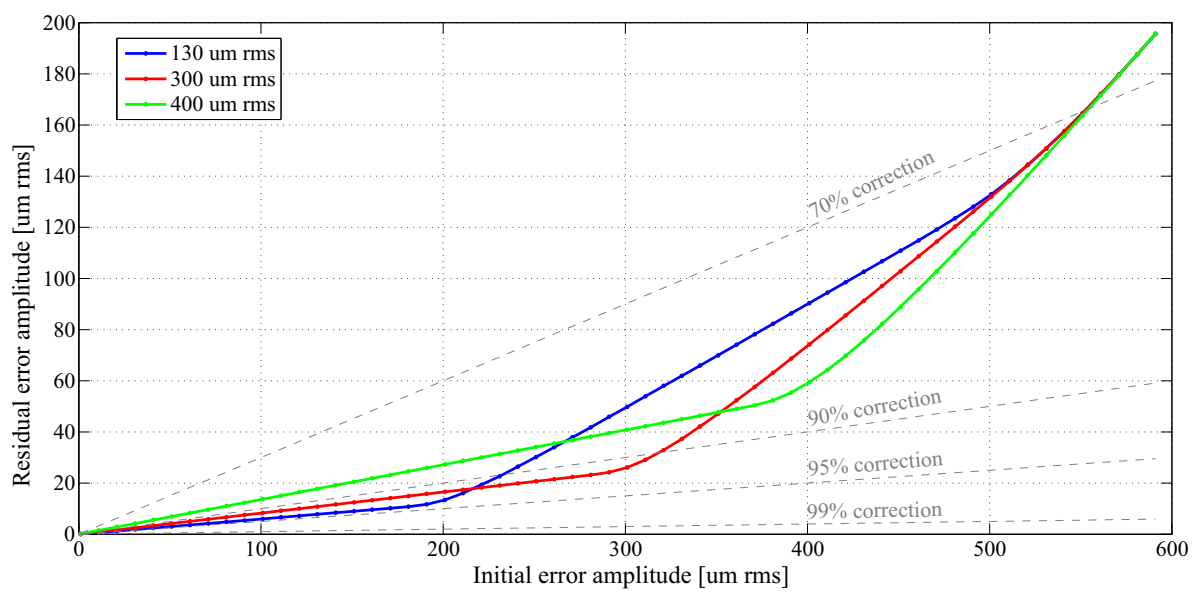

Figure 15. Correction performance of patterns optimized for different initial astigmatism3 error (shown on Fig 14).

If we know in advance that a larger astigmatism3 error will occur, the actuator pattern could directly be optimized for this value. Fig 14 presents electrodes patterns obtained by optimizing the correction of different amplitude of astigmatism3. The edge actuators are always recovered but their size is adapted to accommodate the required amplitude. Bigger actuators will allow to generate a larger deformation before saturation. This result is once again similar to what was observed with the twin-actuator system of the previous method. Both methods are then consistent.

Fig 15 shows the performance of the different patterns over a range of astigmatism3 error. They all present the same behavior, with the 3 functioning domains, but the first saturation is reached for the amplitude specified in the optimization. Each pattern is more efficient than the others on a given range of amplitude. So, the adequate 
pattern can be chosen depending on the required range of correction.

In order to improve the precision of correction, actuators must be added. The pattern can be optimized for different number of actuators (Fig. 16). Although the correction is more efficient with a larger number of actuators, the evolution of the residual error with the number of actuators is not linear. There is an obvious gain going from 8 actuators to 20: the residual error is divided by 3.3. After that the gain is less obvious, the residual error is only divided by 1.7 going from 20 to 52 actuators. The performance of the 52 actuators system is similar to the initial 756 pixel actuators configuration. This shows that it might not be necessary to design a system with a lot of actuators. We can also note that for any numbers of actuators, the edge actuators are always recovered.

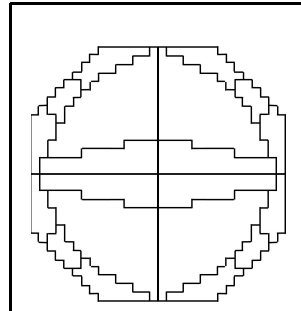

(a)

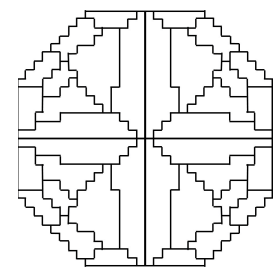

(c)

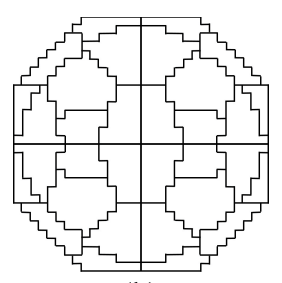

(b)

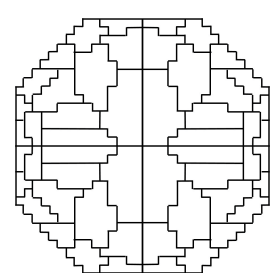

(d)

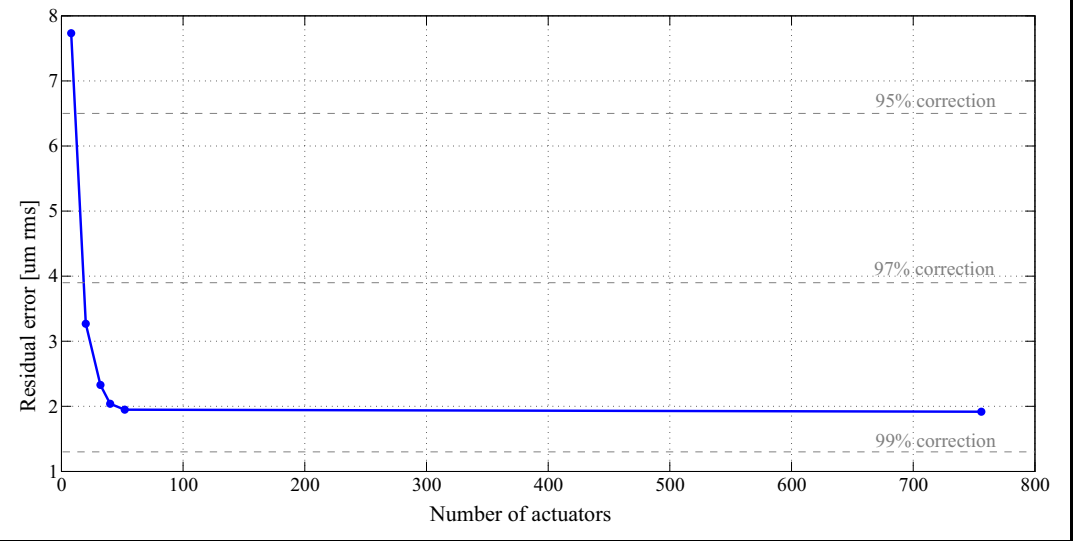

Figure 16. Left: Patterns optimized for a correction of $130 \mu \mathrm{m}$ rms of astigmatism error with different number of actuators: (a) 20 actuators ; (b) 32 actuators ; (c) 40 actuators ; (d) 52 actuators; Right: Evolution of the residual error amplitude (for the correction of $130 \mu \mathrm{m}$ RMS of astigmatism) with the number of actuators (the 8-actuator system is shown in Fig. 14(a), the 20-, 32-, 40- and 52-actuator systems are shown in this figure and the 756-actuator system corresponds to the initial model with pixel actuators).

\subsection{General Example: Correction of 4 Zernike Modes}

We have seen that the initial shape error of the carbon shell mirror is mainly composed of astigmatism3 and that it is possible to design a pattern efficiently correcting for this error. The mirror will also have to correct for other optical errors. For instance, others modes can be present in the initial shape. Or, inserted in an optical instrument, the mirror will be used to compensate for misalignment effects. Additional shape errors can also be induced by fixation devices. The most common aberrations appearing in optical systems are astigmatism3, coma3, spherical3 and trefoil5. ${ }^{17}$ With the same carbon shell mirror model than in the previous Section, we study designs optimized for the correction of all these error modes. Their importance is weighted through their required amplitude.

Considering the shape of the modes, the optimization can still be run on one quarter of the mirror. Note that we have compared the results for optimization run on half the mirror and one quarter: they are similar in terms of performance but there is a considerable gain of time considering only one quarter, as the number of variables is divided by 2 .

As an example, the optimization is run for the correction of $130 \mu \mathrm{m}$ RMS of astigmatism3, $30 \mu \mathrm{m}$ RMS of coma3, $30 \mu \mathrm{m}$ RMS of spherical3 and $10 \mu \mathrm{m}$ RMS of trefoil5. As astigmatism, coma and trefoil can be oriented in different ways, the correction of these modes is optimized for two orthogonal directions.

Fig. 17 shows the optimized patterns for different number of actuators. Edge actuators are once again recovered. 
The optimization is done for a significant amount of astigmatism3, compare to the other modes, so the pattern design is driven by the correction of this mode. We study here the individual mode correction performed with the 40-actuator pattern. As expected, the astigmatism3 error is corrected more efficiently: $97 \%$ of this error is compensated by the system. The trefoil 5 correction is also efficient: $94 \%$ of this error is corrected. This is due to the presence of edge actuators, which are ideal for the correction of this type of modes. However, only $82 \%$ of the coma 3 and spherical3 errors are corrected by this system. But as the required amplitude was low, the amount of residual error is reasonably low for these 2 modes.

Figure 18 shows the evolution of the objective function (Eq. 7) with the number of actuators. As explain before, it corresponds to the amount of residual error if all modes are corrected in the same time. It is interesting to compare this value to what would be achieved by the pattern previously optimized for the correction of $130 \mu \mathrm{m}$ RMS of astigmatism3 only. The new patterns perform significantly better, it shows the interest of optimizing the system according to the expected errors.

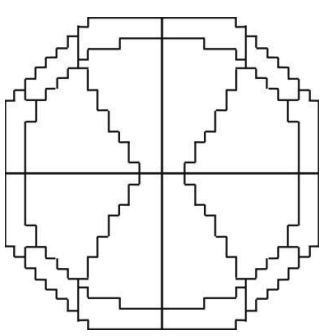

(a)

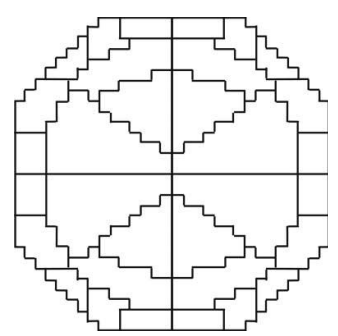

(b)

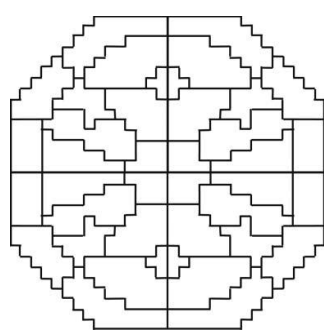

(c)

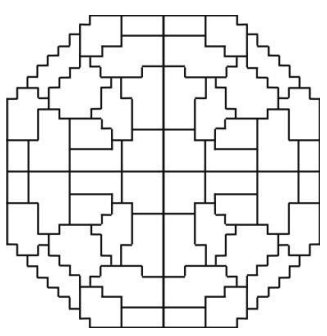

(d)

Figure 17. Patterns optimized for the correction of $130 \mu \mathrm{m}$ RMS of astigmatism3, $30 \mu \mathrm{m}$ RMS of coma3, $30 \mu \mathrm{m}$ RMS of spherical3 and $10 \mu \mathrm{m}$ RMS of trefoil5, with different number of actuators: (a) 20 actuators ; (b) 32 actuators ; (c) 40 actuators ; (d) 52 actuators.

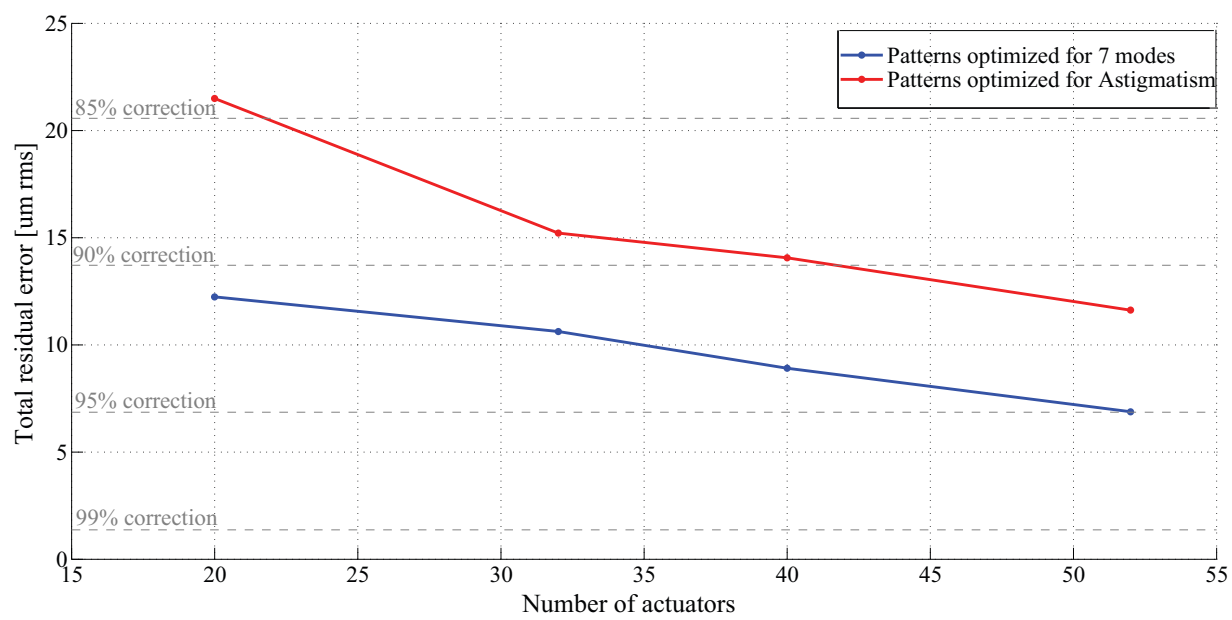

Figure 18. Performance of the different optimized pattern for the correction of a wavefront error of $137 \mu \mathrm{m}$ RMS composed of $130 \mu \mathrm{m}$ RMS of astigmatism3, $30 \mu \mathrm{m}$ RMS of coma3, $30 \mu \mathrm{m}$ RMS of spherical3 and $10 \mu \mathrm{m}$ RMS of trefoil5. The blue curve correspond to patterns directly optimized for this requirement (Fig. 17) and the red curve to patterns optimized only for the correction of $130 \mu \mathrm{m}$ RMS of astigmatism3 (Fig. 16).

In conclusion, we have seen that for a given set of wavefront errors it is possible to design an efficient actuator pattern. The number of actuators can be chosen depending on the required precision of correction. The problem can also be studied in the opposite way: given a number of actuators and a required amount of residual error, what are the different initial errors that can be corrected? The pattern can be optimized for different mode distributions, which will give an information on the errors corrigible by the mirror. This kind of study could 
help to specify the initial shape error of a mirror, constraining more or less the manufacturing tolerances.

\section{CONCLUSION}

Two novel design methods for surface-parallel actuators of ultra-thin mirrors has been presented. Coupling finite element analysis and evolutionnary optimization algorithm, both methods allow to automatically design an actuator pattern given a mirror geometry and correction requirements. The actuation scheme consists of a pattern of electrodes providing an almost full coverage of the piezoelectric layer, and thus maximize the amount of active material that is available for actuation. A set of novel shape optimized patterns was determined, maximizing the system correctability and stroke for a chosen number of independent actuators and for dominant imperfection modes.

The first method was focused on the correction of one single error mode. The starting point was the observation that the correction of a figure error that has at least two planes of mirror symmetry is optimally done with twin actuators that have the same optimized shape but are rotated with respect to each other. For astigmatism3 correction, the resulting optimal design was 4 electrodes system, located on the mirror edge. Once this basic design has been defined, additional sets of optimized twin actuators are defined within the intersection of the previous twin actuators, and an arbitrarily fine actuation pattern can be generated. The second method, more general, considers the correction of several error modes and takes into account actuator saturation directly during the design process. The algorithm starts with a finite element model of the system, composed of numerous small pixel actuators. Once the influences of each pixel actuator are computed, their grouping into bigger actuators is optimized, using Voronoi cells. The resulting patterns present arbitrary, non-intuitive electrode shapes, ideal for the considered correction requirements.

Different actuator arrangements have been compared throughout these studies in order to validate the methods and to highlight the advantages of the different designs. For instance the optimized 41-actuator design resulting from the first method was compared to a 91 actuators design, with hexagonal patches forming a honeycomb layout and to a 92 actuators design, with unidirectional actuators organized in a triangular lattice. Compared to these two classical designs, the optimized design provides a better correctability for the first 25 Zernike modes, despite having less than half the number of actuators. It is particularly the use of small edge electrodes that significantly improves the mirror performance. The same 41-actuator design has also been compared to 40-actuator patterns optimized with the second method. For correction of astigmatism3 only, both methods provide similar results. Considering the correction of several Zernike modes, the best performance is obtained with a pattern optimized with the second method directly for the given set of aberrations.

The excellent performance obtained with the proposed actuator design methods, notably for figure errors dominated by astigmatism3, make them well suited for ultra-thin mirror designs. Of course, the approach presented is applicable to any type of surface-parallel actuated mirror technologies. These methods allow to optimize a deformable mirror design considering a specific application, it will help improving further the optical performance of instruments.

\section{ACKNOWLEDGMENTS}

We thank Xin Ning (Caltech) for help with the optimization algorithm. ML acknowledges the support of a postdoctoral grant from the French Defense procurement agency (DGA) held through Aix-Marseille University, France. Financial support from the Keck Institute of Space Studies and the Dow Resnick Bridge program at Caltech is gratefully acknowledged. A part of this research was carried out at the Jet Propulsion Laboratory, California Institute of Technology, under a contract with National Aeronautics and Space Administration (NASA). 


\section{REFERENCES}

1. F. Roddier, Adaptive optics in astronomy, Cambridge University Press, 1999.

2. R. Wilson, F. Franza, and L. Noethe, "Active optics. I. A system for optimizing the optical quality and reducing the costs of large telescopes.," J. Mod. Opt. 34, pp. 485-509, 1987.

3. N. Lefaudeux, X. Levecq, G. Dovillaire, S. Theis, and L. Escolano, "New deformable mirror technology and associated control strategies for ultrahigh intensity laser beam corrections and optimizations," Society of Photo-Optical Instrumentation Engineers (SPIE) Conference Series 8236, 2012.

4. K. Wang, D. Milkie, A. Saxena, P. Engerer, T. Misgeld, M. Bronner, J. Mumm, and E. Betzig, "Rapid adaptive optical recovery of optimal resolution over large volumes," Nature Methods 11, pp. 625-628, 2014.

5. R. Zawadzki, S. Jones, S. Pilli, D. Kim, S. Olivier, and J. Werner, "Retinal imaging with a combined adaptive optics/optical coherence tomography and adaptive optics/scanning laser ophthalmoscopy system," Society of Photo-Optical Instrumentation Engineers (SPIE) Conference Series 7550, 2010.

6. P. Madec, "Overview of deformable mirror technologies for adaptive optics and astronomy," Society of Photo-Optical Instrumentation Engineers (SPIE) Conference Series 8447, 2012.

7. M. Postman, T. Brown, K. Sembach, M. Giavalisco, W. Traub, K. Stapelfeldt, D. Calzetti, W. Oegerle, M. Rich, P. Stahl, J. Tumlinson, M. Mountain, R. Soummer, T. Hyde, "Advanced Technology LargeAperture Space Telescope: science drivers and technology developments," Optical Engineering 51, p. 011007, 2012.

8. K. Patterson and S. Pellegrino, "Ultralightweight deformable mirrors," Applied Optics 52, pp. 5327-5341, 2013.

9. J. Sinquin, J. Lurcon, and C. Guillemard, "Deformable mirror technologies for astronomy at CILAS," Society of Photo-Optical Instrumentation Engineers (SPIE) Conference Series 7015, 2008.

10. G. Hickey, T. Barbee, M. Ealey, and D. Redding, "Actuated hybrid mirrors for space telescopes," Society of Photo-Optical Instrumentation Engineers (SPIE) Conference Series 7731, 2010.

11. S. Freund, L. Suresh, Thin Film Materials. Stress, Defect Formation and Surface Evolution, Cambridge University Press, 2003.

12. C. Araujo, J. Scott, and G. Taylor, Ferroelectric Thin Films: Synthesis and Basic Properties, Taylor \& Francis, 1996.

13. J. Steeves and S. Pellegrino, "Ultra-Thin Highly Deformable Composite Mirrors," 54th AIAA Structures, Structural Dynamics, and Materials Conference, 2013.

14. Simulia, Abaqus/Standard, version 6.12.

15. T. Coleman and Y. Li, "A Reflective Newton Method for Minimizing a Quadratic Function Subject to Bounds on Some of the Variables," SIAM Journal on Optimization 6, pp. 1040-1058, 1996.

16. R. Noll, "Zernike polynomials and atmospheric turbulence," Journal of the Optical Society of America 66, pp. 207-211, 1976.

17. V. Mahajan, Optical Imaging and Aberrations I: Ray Geometrical Optics, SPIE Press, 1998.

18. N. Hansen, "The CMA evolution strategy: a comparing review," in Towards a new evolutionary computation. Advances on estimation of distribution algorithms, J. Lozano, P. Larranaga, I. Inza, and E. Bengoetxea, eds., pp. 75-102, Springer, 2006.

19. X. Feng, Y. Huang, H. Jiang, D. Ngo, and A. Rosakis, "The effect of thin film/substrate radii on the Stoney formula for thin film/substrate subjected to nonuniform axisymmetric misfit strain and temperature," Journal of Mechanics of Materials and Structures 1, pp. 1041-1053, 2006.

20. F. Aurenhammer, "Voronoi Diagrams A Survey of a Fundamental Geometric Data Structure," ACM Computing Surveys 23, pp. 345-405, 1991.

21. J. Steeves, S. Pellegrino, M. Laslandes, D. Redding, S. Bradford, and T. Barbee, "Design and fabrication of ultra-lightweight active shell mirrors for space telescope applications," Society of Photo-Optical Instrumentation Engineers (SPIE) Conference Series 9151, 2014. 